[To appear in Rev. of Scientific Instruments]

\title{
Frequency Stabilization Of An Extended Cavity Semiconductor Diode Laser For Chirp Cooling
}

\author{
Jacob Morzinski ${ }^{1}$, Parminder S. Bhatia ${ }^{1}$, and M.S. Shahriar ${ }^{1,2}$ \\ ${ }^{1}$ Research Laboratory of Electronics, Massachusetts Institute of Technology, \\ Cambridge, MA 02139. \\ ${ }^{2}$ Department of Electrical and Computer Engineering, Northwestern University, \\ Evanston, IL 60208
}

\begin{abstract}
We present a technique to stabilize to an atomic transition the chirping frequency of a narrow-band semiconductor diode laser. The technique is demonstrated to chirp-cool the ${ }^{85} \mathrm{Rb}$ atoms used for loading a magneto-optical trap. The stabilization process eliminates long term fluctuations and drifts in the number of atoms caught in the trap. This is an extremely simple, easy-to-implement, and robust method for wide range of laser cooling experiments employing frequency chirping.
\end{abstract}


Cooling and trapping of atoms [1] is of considerable current interest. In laser cooling experiments atoms scatter resonant photons from a laser beam directed against the velocity of the atomic beam. The transfer of photon momentum to the atom eventually brings the atom to rest. The primary experimental difficulty in such atom-slowing techniques is the varying Doppler shift experienced by the atom as it slows down. The change in the Doppler shift takes the atom out of resonance with the laser field. Therefore, to keep the atom in resonance with the applied field as it slows down, either the atomic or the laser frequency needs to be smoothly and accurately varied. The technique based on varying the atomic frequency is called Zeeman cooling [2], while the corresponding technique based on varying the laser frequency is called chirp cooling [3-6]. In the latter technique the laser is tuned below the zero-velocity resonance frequency of the moving atom. As the atom slows, the Doppler shifts decrease, and therefore, to keep the atom in resonance, the laser frequency must increase. This is achieved by sweeping (or chirping) the laser frequency as a function of time at a rate equal to the ratio of the Doppler shift at the most probable velocity of the incoming atom to the total stopping time for the atom. To chirp cool the atoms, diode lasers are preferred, not only because of their compactness and low cost [7-9], but also because chirp is easier to achieve with diode lasers. Diode lasers, however, suffer from the frequency drift caused by changes in the junction temperature, by current noise and by the perturbation of the external cavity length. This drift will cause drifting of the chirp, which results in inefficient cooling. Furthermore, it will cause drifting of the end points of the chirp, leading to fluctuations in the final velocity profile of the atomic beam.

This problem can be overcome by using, for example, an acousto-optic modulator (AOM). Briefly, the diode laser frequency is shifted by the AOM, and then locked to an atomic transition using standard methods [10-13]. The AOM driving frequency is then scanned in order to produce the required chirping. One drawback of this approach is that the direction of the output of an $\mathrm{AOM}$ is correlated with the driving frequency, so that the 
scan is accompanied by undesired angular variations. One can overcome this problem by using a compensating, scanning mirror. However, in order for this process to work well, the scan range should be a small fraction of the base frequency. Given that the scan range is typically a few hundred $\mathrm{MHz}$, this implies that the AOM to be used should have a base frequency in the $\mathrm{GHz}$ range. An $\mathrm{AOM}$ at such a high frequency is generally expensive, and not very efficient. As such, only a small fraction of the total diode laser power is available for chirp cooling. In this paper, we demonstrate an alternative method that does not require an AOM, and makes most of the diode laser power available for cooling. It is simple, robust, and requires only inexpensive electronics.

In our experimental setup shown in Fig. 1 the atomic beam consists of rubidium atoms which effuse from a small hole in an oven operating at $\approx 250^{\circ} \mathrm{C}$. This atomic beam is collimated and is finally directed into a vacuum chamber evacuated to about $10^{-10}$ Torr. The most probable velocity of the ${ }^{85} \mathrm{Rb}$ atoms in the atomic beam is $\approx 320 \mathrm{~m} / \mathrm{sec}$ which corresponds to the Doppler shift of $\approx 680 \mathrm{MHz}$. To chirp cool the atoms in this atomic beam we use a commercially adopted version (Tui Optics: DL 100) of the extended-cavity diode laser described earlier by Hansch and co-workers [8]. This system uses a solitary laser diode (HL $7857 \mathrm{G}$ ) which operates near $780 \mathrm{~nm}$. When free running, this laser operates in multimode with broad bandwidth. In this laser system, external feedback to the laser diode provided by a grating in the Littrow configuration forces the laser to operate in single mode with a linewidth of about $1 \mathrm{MHz}$ [9]. The coarse tuning of the laser output wavelength is achieved by changing the junction temperature and the injection current of the laser diode, while the fine tuning is achieved by changing the tilt angle of the diffraction grating with a piezo actuator. This diode laser beam is overlapped with a second repump laser beam. The two superimposed beams are circularly polarized and focused inside the vacuum chamber, where they overlap and propagate counter to the atomic beam. Inside the vacuum chamber there is about $10 \mathrm{~mW}$ of diode laser power with a beam diameter of $5 \mathrm{~mm}$, and about $75 \mathrm{~mW}$ of repump laser power with a beam diameter 
of $1 \mathrm{~cm}$. As shown in Fig. 2, the diode laser is tuned to the $5 S_{1 / 2}(F=3) \rightarrow 5 P_{3 / 2}\left(F^{\prime}=4\right)$ transition of the ${ }^{85} \mathrm{Rb}$ atom. The diode laser beam decelerates the ${ }^{85} \mathrm{Rb}$ atoms, which are finally brought to rest. The total stopping distance in our experiment is about one meter. The function of the epump beam is to prevent the optical pumping of the atoms into the lower hyperfine level $5 S_{1 / 2}(F=2)$ of the ground state (Fig. 2) [14].

A block diagram of the locking circuit is shown in Fig. 3. The diode laser frequency is swept by a voltage ramp from the laser controller, which is applied to the grating drive mechanism of the diode laser. The absorption spectrum, which lies within the frequency sweep of the laser, is monitored with a photodiode. The signal from the photodiode is differentiated electronically. The differentiated signal is then multiplied by the output of the pulse generator, which generates TTL pulses with adjustable time delay. The output of the multiplier is fed to an integrator. The integrated output is the feedback signal, which is added to the ramp voltage that drives the grating to sweep the laser frequency.

To lock the sweeping laser frequency, the time delay of the pulse from the pulse generator is adjusted so that the pulse coincides with the zero-crossing point of the differentiated signal (Fig. 4). Under this condition the error signal is zero. Any drift in the laser output frequency results in an offset between the pulse from the pulse generator and the zero-crossing point of the differentiated signal. This results in a nonzero value of the error signal, whose magnitude and sign are determined by that of the offset. Fig. 5 shows the schematic of the actual circuit. The complete circuit consists of four essential parts viz. (1) differentiator (2) timer (3) integrator (4) adder.

Fig. 6(b) shows a typical signal as experimentally observed through the absorption cell containing ${ }^{85} \mathrm{Rb}$ atoms. This signal was observed using the ramp voltage shown in Fig. 6 (a). Fig. 6(c) shows the output of the differentiator when the signal shown in Fig. 6(a) was supplied to its input. In Fig. 6 the peak position of the signal and the zerocrossing point of the differentiated signal are coincident with the pulse from the pulse 
generator shown in Fig 6(d). Some kinks in Fig. 6(b) and (c) are due to the presence of the neighboring hyperfine transitions shown in Fig. 2, which lies within the Doppler envelope and complicates the interpretation of the observed spectrums.

In a real experiment chirp cooled atoms are loaded into the magneto-optical trap (MOT), where three pairs of counterpropagating laser beams subsequently trap them. In our experiment, with the feedback loop shown in Fig. 3 closed, we typically achieve a stable trap with approximately ten million atoms. As the feedback loop is opened, the trap get weaker and finally disappears, illustrating the potential importance of using laser stabilization during chirp cooling.

In conclusion, we have presented a technique and its implementation to stabilize the chirping frequency of a diode laser. The technique is simple and robust, requiring only simple electronics. This stabilization process eliminates long term fluctuations and drifts in the number of atoms caught in a magneto-optic trap, for example.

\section{$\underline{\text { Acknowledgements }}$}

This work was supported by AFOSR grant \# F49620-98-1-0313 and ARO grant \#s DAAG55-98-1-0375 and DAAD19-00-1-0177 


\section{References}

[1] Harold J. Metcalf, Peter van der Straten, " Laser Cooling and Trapping", Springer, 1999.

[2] J. Prodan, A. Migdall, W. D. Phillips, I. So, H. Metcalf, and J. Dalibard, " Stopping atoms with laser light ", Phys. Rev. Letts. 54992 (1985).

[3] W. Ertmer, R. Blatt, J. L. Hall, and M. Zhu, " Laser manipulation of atomic beam velocities", Phys. Rev. Lett. 54, 996 (1985).

[4] R. N. Watts, C. E. Wieman, " Manipulating atomic velocities using diode lasers", Opt. Letts. 11291 (1986).

[5] C. C. Bradley, J. G. Story, J. J. Tollett, J. Chen, N. W. M. Ritchie, and R. G. Hulet, " Laser cooling of lithium using relay chirp cooling", Opt. Lett. 17, 349 (1992).

[6] V. I. Balykin, V. S. Letokhov, and V. I. Mushin, JETP Lett. 29. 560 (1979). 
[7] C. E. Wieman, L. Hollberg, “Using diode lasers for atomic physics”, Rev. Sci. Instrum. 62 (1991) 1- 20.

[8] L. Ricci, M. Weidemuller, T. Esslinger, A. Hemmerich, C. Zimmermann, V. vuletic, W. Konig, T.W. Hansch , “ A compact grating-stabilized diode laser system for atomic physics", Opt. Commun. 117 (1995) 541-549.

[9] Parminder S. Bhatia, George R. Welch, and M. O. Scully, " A single mode semiconductor diode laser operating in strong feedback regime and tunable within the $D_{1}$ line of Cs atom", Opt. Commu. 189 (2001) 321-336.

[10] M. de Labachelerie and P. Cerez, " Frequency locking of a $850 \mathrm{~nm}$ external-cavity semiconductor laser on a Doppler free Cs-D2 line, " Proc. SPIE 701, 182-184 (1986).

[11] T.P. Dinneen, C.D. Wallace and P.L. Gould, " Narrow linewidth, highly stable, tunable diode laser system", Opt. Commun. 92 (1992) 277-282.

[12] M. de Labachelerie, C. Latrasse, K. Diomande, P. Kemssu, and P. Cerez, " A $1.5 \mu m$ absolutely stabilized external cavity semiconductor laser", IEEE Trans. Instr. Meas. 40, 185-190( 1991).

[13] Y. Millerioux, D. Touahi, L. Hilico, A. Clairon, R. Felder, F. Biraben, and B. de Beauvoir, " Towards an accurate frequency standard at $780 \mathrm{~nm}$ using a laser diode stabilized on a hyperfine component of Doppler free two photon transition in Rubidium", Opt. Commun. 108, 91-96 (1994). 
[14] C. D. Wallace, " Measurement of trap loss rate coefficients and trap characterstics of Rubidium magneto-optical trap", Ph. D. thesis, (University of Connecticut 1994).

\section{Figure Captions}

Fig. 1: Shows the experimental apparatus employed for the chirp cooling. UHV: Ultra high vacuum, $\lambda$ /4: Quarter wave plate, P: Polarizer, BS: Beam splitter, L1, L2 Lenses, M: turning mirror.

Fig. 2: Energy level splitting of $R b^{85}$. Chirp and repump lasers are tuned to the transitions with label 1 and 2 respectively.

Fig. 3: Block diagram of the electronic circuit used to stabilize the chirp laser.

Fig. 4: (a) Absorption signal (b) differentiated signal (c) pulse from pulse generator. In this figure $t_{0}$ is the zero crossing point of the differentiated signal.

Fig. 5: Schematic of the electronic circuit.

Fig. 6: (a) Voltage sweep (b) absorption signal (c) differentiated signal (d) pulse from pulse generator. 


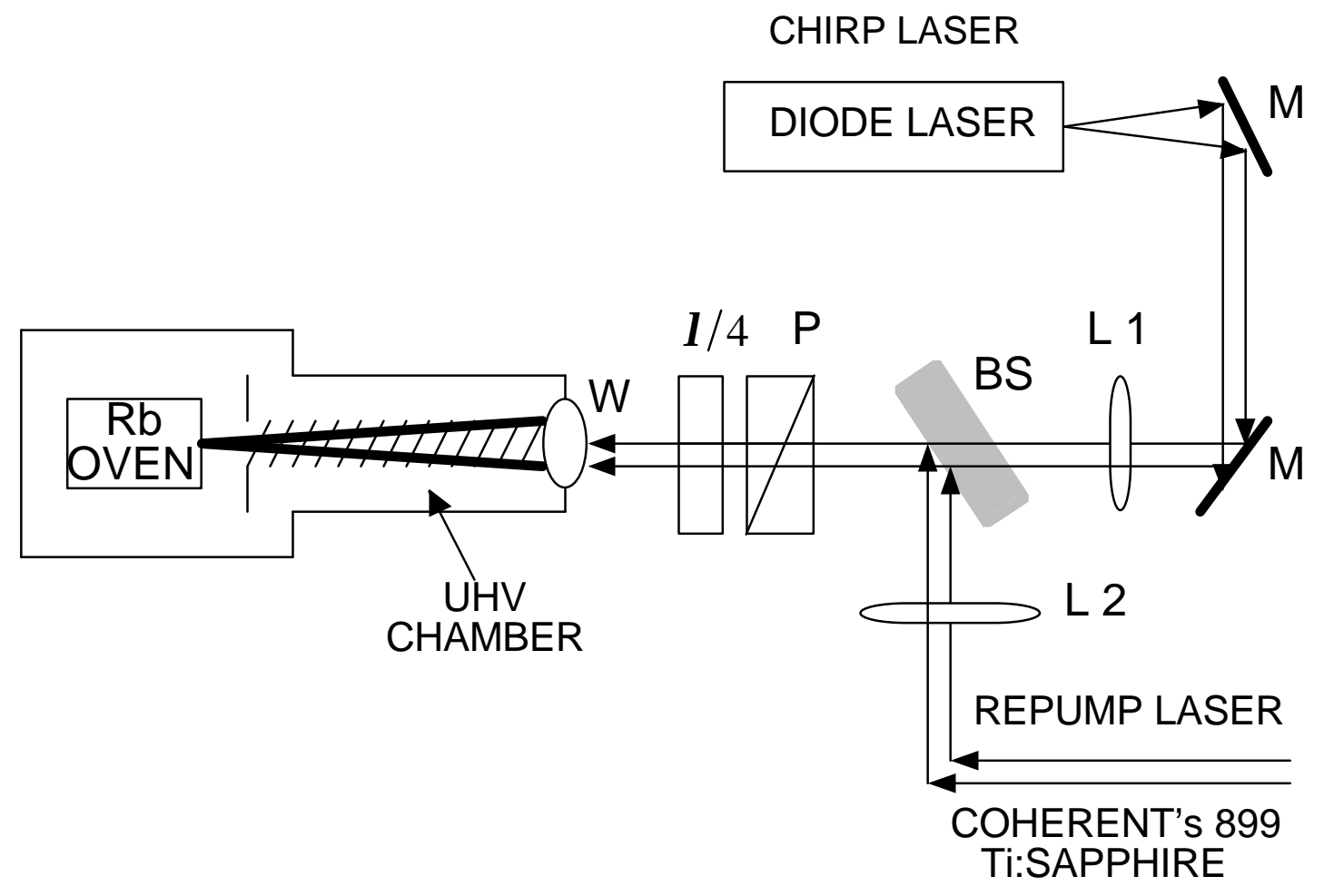

FIGURE 1. 
$F^{\prime}$

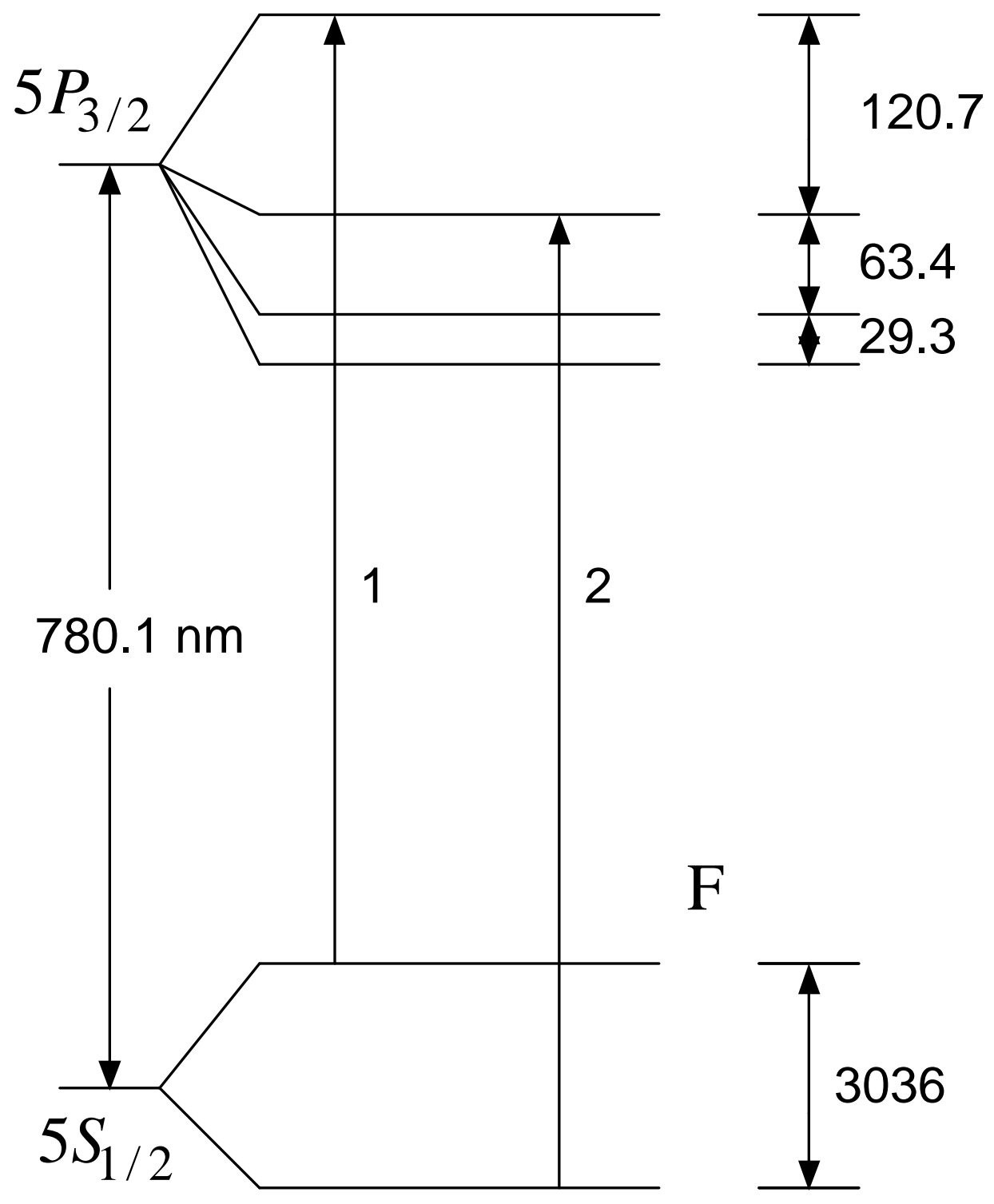

FIGURE 2. 


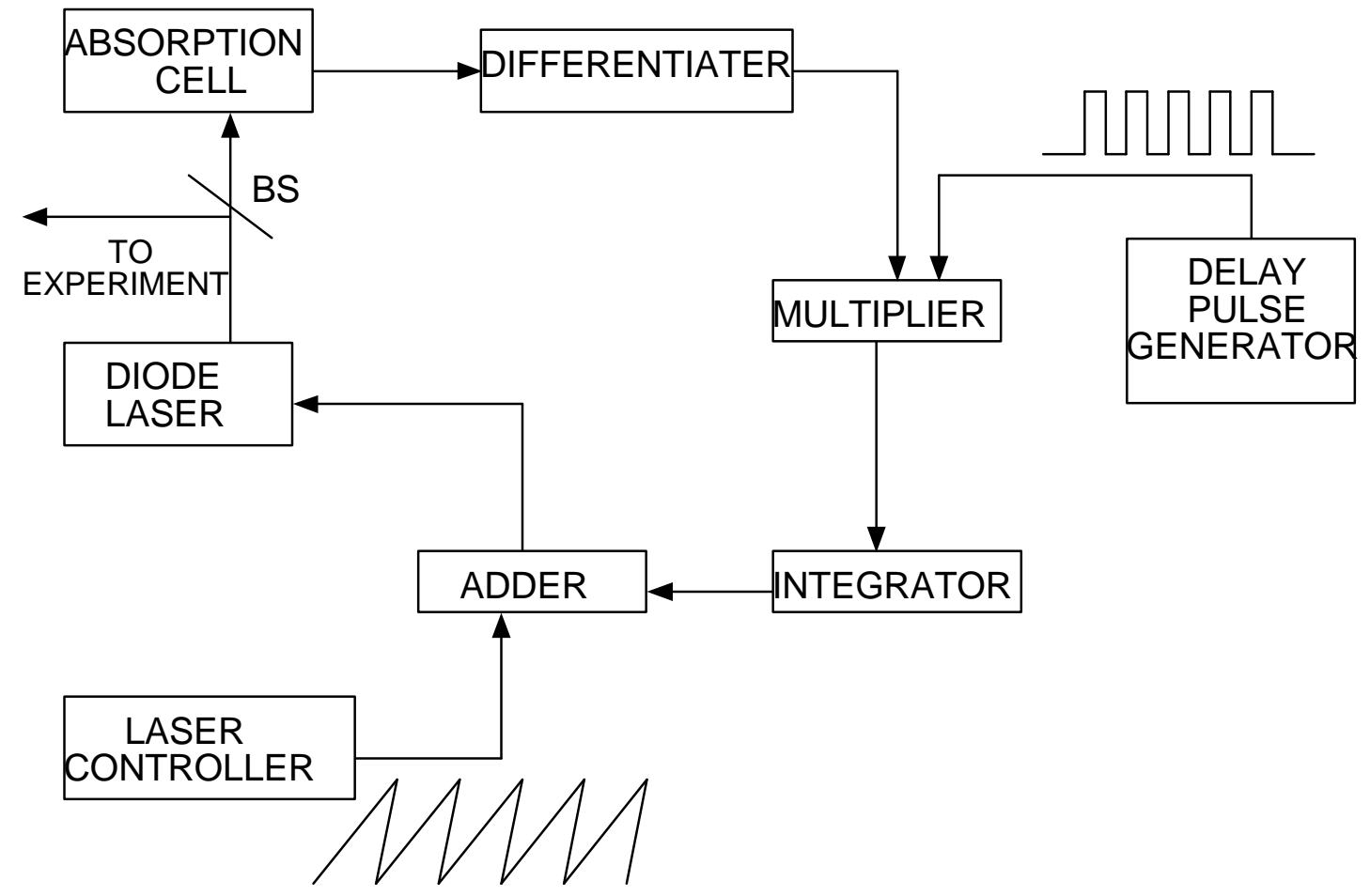

FIGURE 3. 


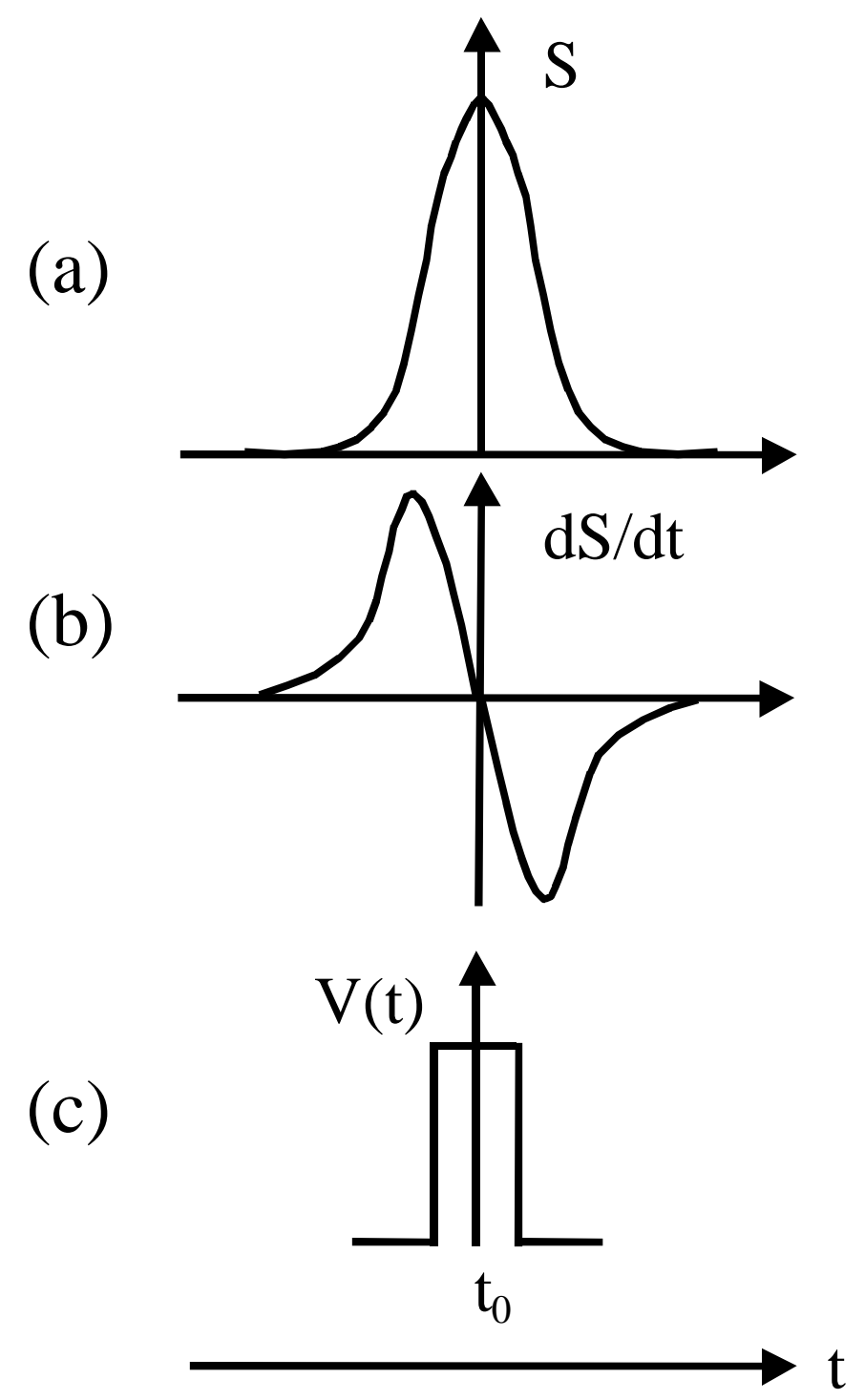

FIGURE 4. 

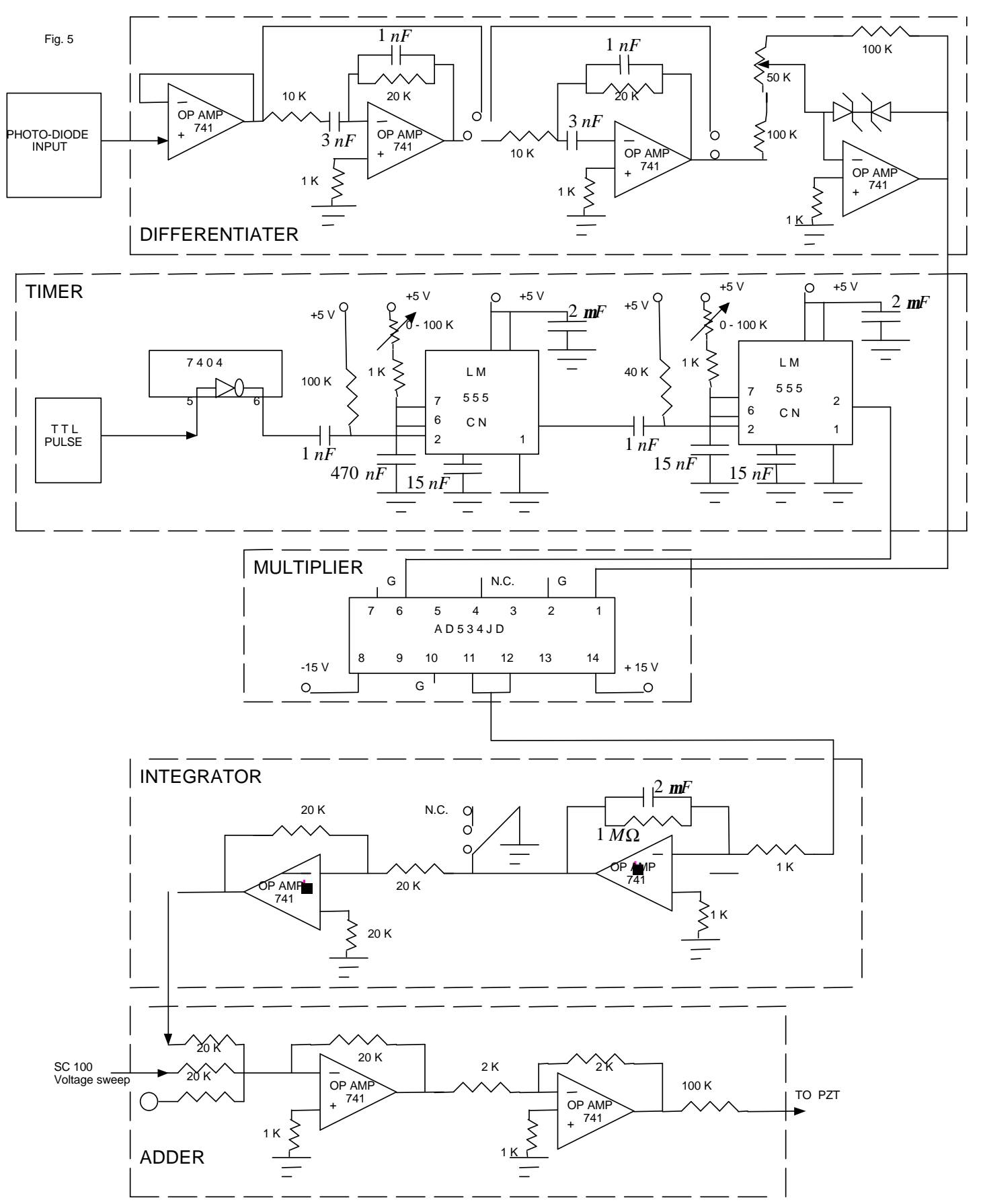

FIGURE 5. 


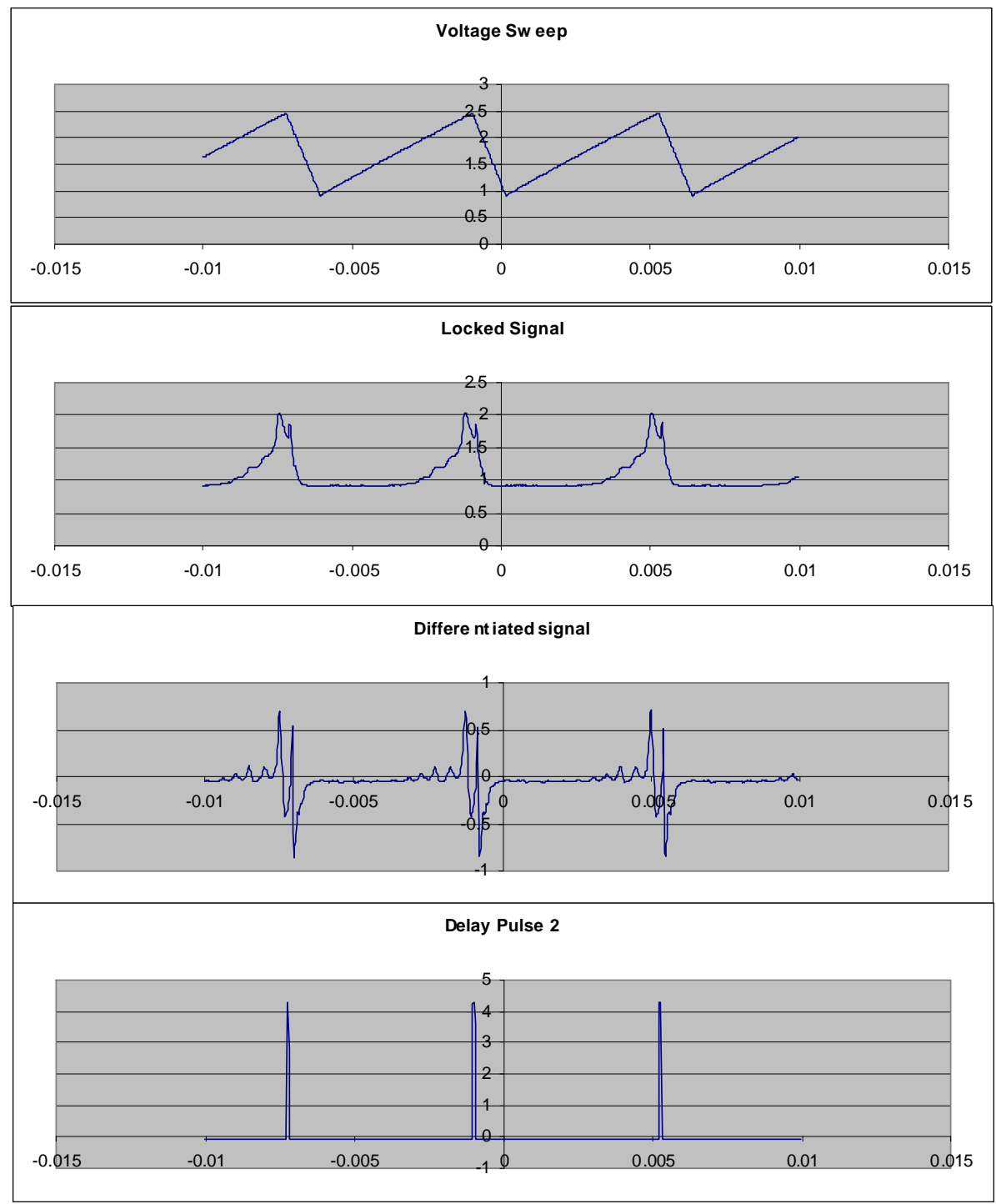

FIGURE 6. 